\title{
A theoretical model to embrace physical activity counselling in primary care practice
}

\section{Vanitha Arumugam and Joy C. MacDermid}

\section{University of Western Ontario}

\section{Introduction}

Canadian guidelines for physical activity recommend 150 minutes of moderate physical activity every week in cycles lasting at least 10 minutes. ${ }^{1}$ According to a state report, $85 \%$ of Canadians do not achieve the recommended guidelines for physical activity, $^{2}$ posing a great risk for obesity and chronic non-communicable diseases like cardiovascular disease, diabetes, osteoporosis and cancer. Physical activity counselling (PAC) is a proven means to promote physical activity, ${ }^{3}$ for which a $10 \%$ increase could save up to $\$ 150$ million per annum in direct healthcare related costs in Canada. Importantly, primary care providers

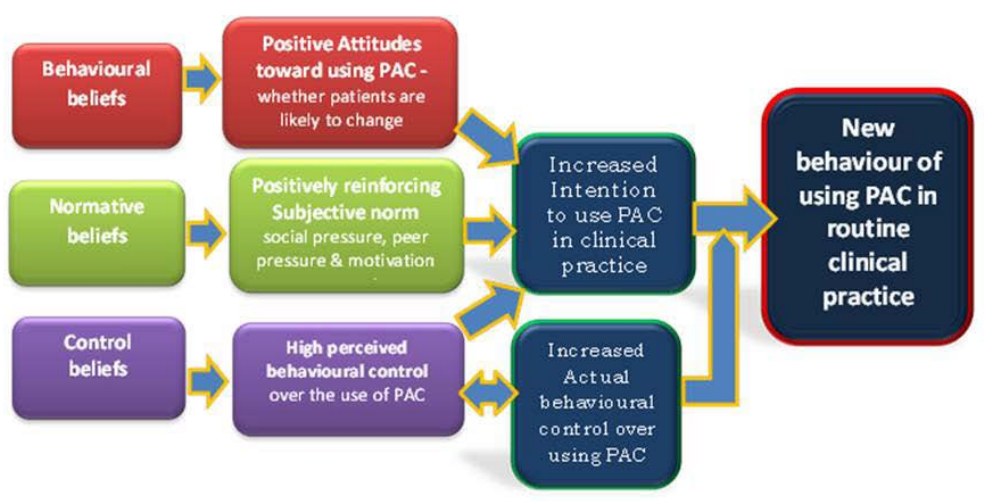
are in a unique position to influence physical activity through healthy lifestyle recommendations. The purpose of this paper is to describe a theoretical model to understand how PAC can be implemented in the routine practice of primary care physicians.

On average, Canadians make approximately 16.2 million family physician visits every year, ${ }^{4}$ and are the first point of contact with the health care system. As such this provides a convenient opportunity for physicians to deliver PAC, and effect a positive change over other healthcare providers. Delivering PAC in clinical practice is essential to improve level of fitness in patients who have suboptimal levels of physical activity, as most patients believe that primary care physicians are their source for preventive health information and recommendations. ${ }^{5}$ However the amount of PAC delivered by primary care physicians to patients varies greatly. ${ }^{6}$

Physicians have identified multiple barriers to providing PAC, including a lack of time available to counsel during

questions about whether it changes the behaviour of their clients, as well as discomfort in providing advice due to lack of training and little or no reimbursement for preventive measures.?

In order to understand how physician behaviour can be modified to use PAC in practice, theoretical models can be useful to clarify the internal processes involved in behavioural change. This can be particularly helpful for policy makers to ascertain how physician behaviour can adapt to embrace new policies or procedures. ${ }^{8}$

\section{Theoretical model}

A key model that can help policy and decision makers understand the process of implementing PAC in primary care practice is the 'Theory of Planned Behaviour' (See Figure 1), which suggests that planning and preparation will facilitate the adoption of a specific behaviour. ${ }^{9}$ The key elements of this theory are: a) Behavioural beliefs: These are individual beliefs about the consequence of a behaviour; - 
b) Normative beliefs: These are individual perceptions of social pressures to perform a behaviour; $c$ ) Control beliefs: These are individual beliefs about the factors that facilitate or impede performance of a behaviour. ${ }^{10}$ Behavioural beliefs affect attitudes towards a behaviour, normative beliefs lead to subjective norms and control beliefs result in perceived behavioural control. At the next level, when the attitude towards a behavioural belief and subjective norm is favourable along with a greater perceived control of the behaviour, intention to perform a behaviour increases greatly. An increase in perceived feeling of actual control over a behaviour along with a strong intention would result in action when the opportunity arises. ${ }^{9}$

\section{Model for PAC implementation}

In this model, the behavioural beliefs are whether primary care physicians deem administering PAC to patients to be effective in modifying patient behaviour. Physicians' behavioural beliefs vary on whether they think physical activity can affect health, or whether their clients are willing to initiate or improve their activity levels. ${ }^{7}$ Normative beliefs can affect behaviours as physicians may be influenced by opinion leaders' attitudes about PAC and also organisational policies. Finally, control beliefs are the perceived beliefs regarding ability to implement PAC which might include decision-control, policy or environmental restrictions around PAC. A positive attitude towards PAC and its effects on patients' physical activity levels as well as subjective norms along with high perceived control over the implementation of PAC would increase the intentions of primary care physicians to embrace PAC. Consequently, when the right opportunities are presented to primary care physicians, such as a clinic visit by a patient who will benefit from PAC, or an amenable work environment, they will be more likely to embrace PAC in routine clinical practice. ${ }^{8}$

\section{Final remarks}

With so much of evidence to support the use of PAC in improving physical activity in patients, it is important for policy and decision makers to understand the need to work from the bottom-up, starting from the actual attitudes of physicians, subjective norms and perceived control over behaviour. This can be achieved by enacting laws and clinical guidelines alongside incentives to encourage this culture of incorporating PAC into routine clinical practice.
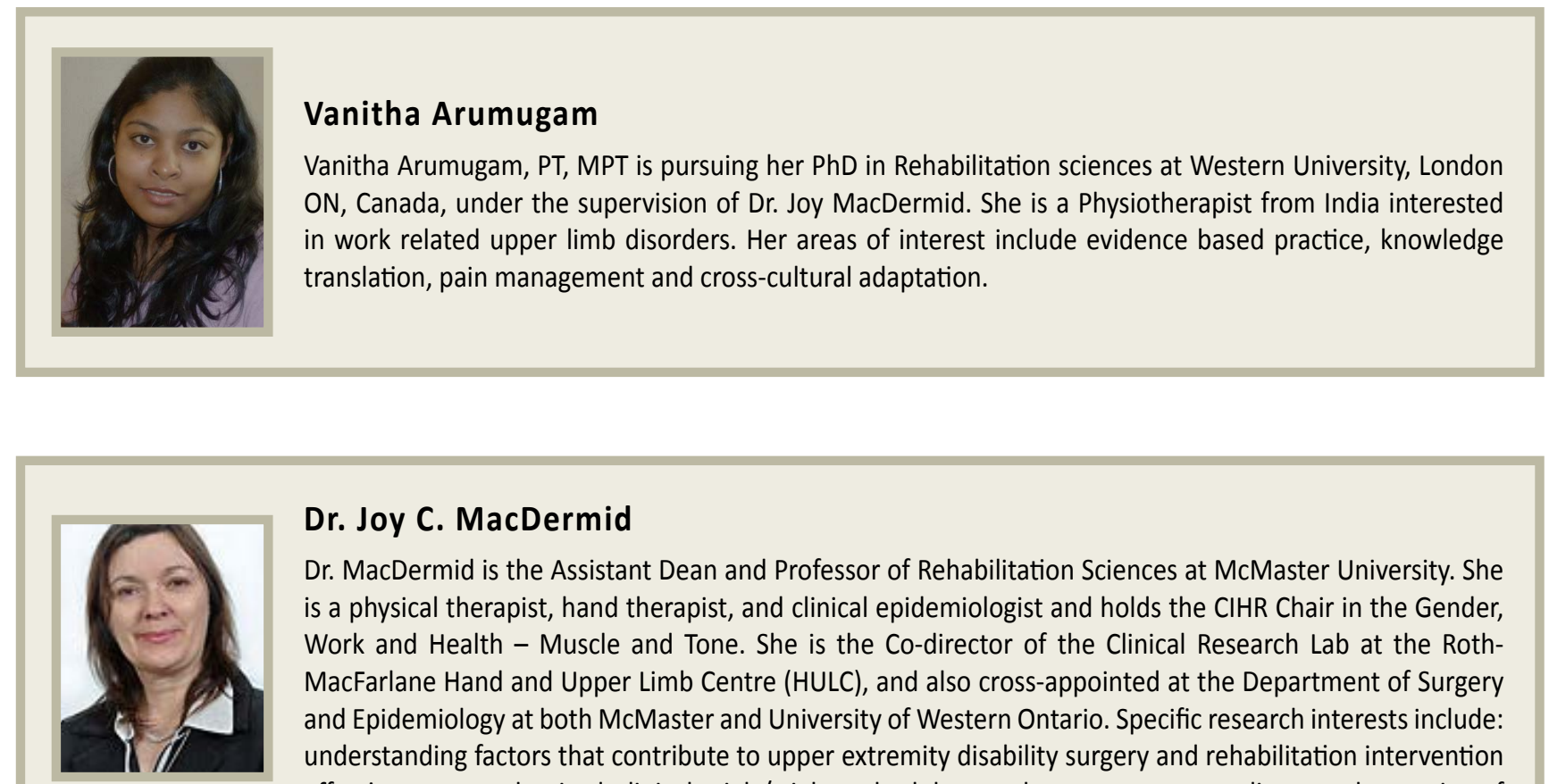

\section{Dr. Joy C. MacDermid}

Dr. MacDermid is the Assistant Dean and Professor of Rehabilitation Sciences at McMaster University. She is a physical therapist, hand therapist, and clinical epidemiologist and holds the CIHR Chair in the Gender, Work and Health - Muscle and Tone. She is the Co-director of the Clinical Research Lab at the RothMacFarlane Hand and Upper Limb Centre (HULC), and also cross-appointed at the Department of Surgery and Epidemiology at both McMaster and University of Western Ontario. Specific research interests include: understanding factors that contribute to upper extremity disability surgery and rehabilitation intervention effectiveness, randomized clinical trials/trial methodology, cohort outcomes studies, psychometrics of clinical measurement (performance or self-report, measures of pain/disability/quality-of-life), clinical epidemiology, clinical practice guidelines, and knowledge transfer. Courses developed and taught include upper extremity clinical skills research and evidence-based practice, quality-of-life (measurement/ research), knowledge exchange, and transfer. 


\section{References}

1. Tremblay MS, Warburton DE, Janssen I, Paterson DH, Latimer AE, Rhodes RE, et al. New Canadian physical activity guidelines. Appl Physiol Nutr Metab. 2011 Feb;36(1):36-46; 47-58.

2. Katzmarzyk PT, Gledhill N, Shephard RJ. The economic burden of physical inactivity in Canada. CMAJ. 2000 Nov 28;163(11):1435-1440.

3. Katzmarzyk PT. The health economics of physical inactivity in Canada. 2011;4(March):30-31.

4. Canadian collaborative centre for physician resources. Family physician shortage estimates [internet]. 2009. Available from: http://www.cma.ca/ multimedia/CMA/Content_Images/Policy_Advocacy/Policy_Research/10FP_Shortage-E.pdf.

5. Stott NCH, Pill RM. Advise yes, dictate no. Patients' views on health promotion in the consultation. Fam Pract. 1990 June 1;7(2):125-131.

6. Florindo AA, Mielke GI, Gomes GA, Ramos LR, Bracco MM, Parra DC, et al. Physical activity counseling in primary health care in Brazil: A national study on prevalence and associated factors. BMC Public Health. 2013 Aug 31;13:794-2458-13-794.

7. Wechsler H, Levine S, Idelson RK, Rohman M, Taylor JO. The physician's role in health promotion: A survey of primary-care practitioners. N Engl J Med. 1983;308(2):97-100.

8. Haines A, Rogers S. Integrating research evidence into practice. In: Silagy C, Haines $A$, editors. Evidence-based practice in primary care. 2nd ed. London: BMJ publishing group; 2001. p. 157-174.

9. Haines A, Kuruvilla S, Borchert M. Bridging the implementation gap between knowledge and action for health. World Health Organization. Bulletin of the World Health Organization. 2004 Oct;82(10):724-31; discussion 732.

10. Ajzen I. The theory of planned behavior. Organ Behav Hum Decis Process. 1991;50(2):179. 